\title{
FIXED POINTS FOR DISCONTINUOUS QUASIMONOTONE MAPS IN SEQUENCE SPACES
}

\author{
SABINA SCHMIDT
}

(Communicated by Palle E. T. Jorgensen)

\begin{abstract}
In [2] Hu gives a fixed point theorem for discontinuous quasimonotone increasing maps in $X=\mathbb{R}^{n}$. We will answer the question in [2] as to whether this result can be extended to $X=l^{p}, 1 \leq p \leq \infty$.
\end{abstract}

Let the Banach spaces $X=\mathbb{R}^{n}, c_{0}$, and $l^{p}, 1 \leq p \leq \infty$, be ordered by the cone $K=\left\{x=\left(x_{i}\right)_{i \in I} \in X: x_{i} \geq 0\right\}$, where $I=\{1, \ldots, n\}$ or $I=\mathbb{N}$, respectively. Then $(X, K)$ is order complete, i.e., if a subset $A$ of $X$ has an upper bound, then $A$ has a least upper bound, which we denote by $\sup A$.

For $u, v \in X, u \leq v$, we set $[u, v]=\{z \in X: u \leq z \leq v\}$. It is well known (theorem of Tarski; see [4]) that for each increasing map $M:[u, v] \rightarrow[u, v]$ the points $\bar{x}=\sup \{x \in[u, v]: x \leq M x\}$ and $\underline{x}=\inf \{x \in[u, v]: x \geq M x\}$ are fixed points of $M$.

For a function $f=\left(f_{i}\right)_{i \in I}: X \rightarrow X$ we define for $x \in X$, in analogy to the notation in [2],

$$
\begin{aligned}
& D_{ \pm} f_{i}(x)=\liminf _{t \rightarrow 0 \pm} \frac{1}{t}\left(f_{i}\left(x+t e^{i}\right)-f_{i}(x)\right), \\
& D^{ \pm} f_{i}(x)=\limsup _{t \rightarrow 0 \pm} \frac{1}{t}\left(f_{i}\left(x+t e^{i}\right)-f_{i}(x)\right),
\end{aligned}
$$

where $e^{i}, i \in I$, are the elements of $X$ with components $e_{j}^{i}=1$ for $i=j$, $e_{j}^{i}=0$ for $i \neq j$.

Theorem. Let $u, v \in X, u \leq v$, and $f=\left(f_{i}\right)_{i \in I}:[u, v] \rightarrow X$ be a function with the following properties:

$$
u_{i} \leq f_{i}\left(x+\left(u_{i}-x_{i}\right) e^{i}\right), \quad v_{i} \geq f_{i}\left(x+\left(v_{i}-x_{i}\right) e^{i}\right) \quad \text { for } x \in[u, v], \quad i \in I .
$$

Then $f$ has a greatest fixed point $\bar{x}$ and a smallest fixed point $\underline{x}$, and

$$
\bar{x}=\sup \{x \in[u, v]: x \leq f(x)\}, \quad \underline{x}=\inf \{x \in[u, v]: x \geq f(x)\} .
$$

Received by the editors July 26, 1990 and, in revised form, November 27, 1990.

1980 Mathematics Subject Classification (1985 Revision). Primary 47H10, 47H05. 
In case $X=\mathbb{R}^{n}$ this result is exactly [2, Theorem 2].

Proof. For each $x \in X, i \in I$ define

$$
g_{i}^{x}(t)=f_{i}\left(x+\left(t-x_{i}\right) e^{i}\right) \quad \text { for } t \in\left[u_{i}, v_{i}\right] .
$$

From (2) and (3) we get $\min \left\{D_{-} g_{i}^{x}(t), D_{+} g_{i}^{x}(t)\right\}>-\infty$ for $t \in\left[u_{i}, v_{i}\right]$ and $u_{i} \leq g_{i}^{x}\left(u_{i}\right), v_{i} \geq g_{i}^{x}\left(v_{i}\right)$.

Hence by [2, Corollary 1] the function $g_{i}^{x}(t)$ has a greatest fixed point $M_{i} x$ and a smallest fixed point $m_{i} x$ and

$$
\begin{aligned}
& M_{i} x=\sup \left\{t \in\left[u_{i}, v_{i}\right]: t \leq g_{i}^{x}(t)\right\}, \\
& m_{i} x=\inf \left\{t \in\left[u_{i}, v_{i}\right]: t \geq g_{i}^{x}(t)\right\} .
\end{aligned}
$$

Since, by (1), $g_{i}^{x}(t) \leq g_{i}^{y}(t)$ for each pair $x, y \in[u, v]$ with $x \leq y$, we conclude that $M_{i} x \leq M_{i} y, m_{i} x \leq m_{i} y$ for $x, y \in[u, v]$ with $x \leq y$. Therefore $M x=\left(M_{i} x\right)_{i \in I}$ defines an increasing map $M:[u, v] \rightarrow[u, v]$.

Let $\bar{x}=\sup \{x \in[u, v]: x \leq M x\}$ be the greatest fixed point of $M$. Combining the equations $\bar{x}_{i}=M_{i} \bar{x}$ and $g_{i}^{\bar{x}}\left(M_{i} \bar{x}\right)$, we get $\bar{x}_{i}=g_{i}^{\bar{x}}\left(\bar{x}_{i}\right)=f_{i}(\bar{x})$ for each $i \in I$. Therefore $\bar{x}$ is also a fixed point of $f$. Since $x \leq f(x)$ implies $x \leq M x$ by (5), we claim $x \leq \bar{x}$ and hence $\bar{x}=\sup \{x \in[u, v]: x \leq f(x)\}$.

In a similar way we can prove that the smallest fixed point $\underline{x}$ of the increasing map $m:[u, v] \rightarrow[u, v]$ is the smallest fixed point of $f$ and that the second equation in (4) is fulfilled.

Corollary. Let $u, v \in X, u \leq v$, and $f=\left(f_{i}\right)_{i \in I}:[u, v] \rightarrow X$ be a function with the following properties:

$$
\begin{gathered}
f_{i}(x) \geq f_{i}(y) \quad \text { for } x, y \in[u, v] \text { with } x \leq y \text { and } x_{i}=y_{i}, \quad i \in I ; \\
\max \left\{D^{-} f_{i}(x), D^{+} f_{i}(x)\right\}<\infty \text { for } x \in[u, v], \quad i \in I ; \\
u_{i} \geq f_{i}\left(x+\left(u_{i}-x_{i}\right) e^{i}\right), \quad v_{i} \leq f_{i}\left(x+\left(v_{i}-x_{i}\right) e^{i}\right) \quad \text { for } x \in[u, v], \quad i \in I .
\end{gathered}
$$

Then $f$ has a greatest fixed point $\bar{x}$ and a smallest fixed point $\underline{x}$ and

$$
\bar{x}=\sup \{x \in[u, v]: x \geq f(x)\}, \quad \underline{x}=\inf \{x \in[u, v]: x \leq f(x)\} .
$$

Remarks. (1) In infinite-dimensional Banach spaces $X$ a function $f: X \rightarrow X$ is quasimonotone increasing if $x, y \in X, x \leq y, \varphi \in K^{*}, \varphi(x)=\varphi(y)$ implies $\varphi(f(x)) \leq \varphi(f(y))$, where $K^{*}=\left\{\varphi \in X^{*}: \varphi(x) \geq 0\right.$ for all $\left.x \in K\right\}$ (see Volkmann [3]). For $X=\mathbb{R}^{n}, c_{0}$, or $l^{p}, 1 \leq p<\infty$, condition (1) is the same as quasimonotonicity. In case $X=l^{\infty}$ condition (1), even together with (2), is weaker than quasimonotonicity. Choose $\varphi \in K^{*}$ with $\varphi(x)=0$ for $x \in c_{0}$ and $\varphi(e)=1$ for $e=\left(e_{n}\right)_{n \in \mathbb{N}}$ with $e_{n}=1$ for all $n \in \mathbb{N}$. For $x=\left(x_{n}\right)_{n \in \mathbb{N}} \in l^{\infty}$, define

$$
f_{n}(x)= \begin{cases}1 & \text { for } x_{n} \leq 0, \\ 1-n x_{n} & \text { for } 0 \leq x_{x} \leq \frac{1}{n}, \\ 0 & \text { for } x_{n} \leq \frac{1}{n},\end{cases}
$$

Then $f=\left(f_{n}\right)_{n \in \mathbb{N}}: l^{\infty} \rightarrow l^{\infty}$ satisfies (1) and (3), but is not quasimonotone increasing.

(2) In $X=l^{\infty}$, methods analogous to these used in this paper also lead to existence theorems for ordinary differential equations with quasimonotone right-hand side (see [1]). 


\section{REFERENCES}

1. A. Chaljub-Simon and P. Volkmann, Un théorème d'existence et de comparison pour des équations différentielles dans les espaces de fonctions bornées, C.R. Acad. Sci. Paris Ser. I Math. (to appear).

2. S.-C. Hu, Fixed points for discontinuous quasi-monotone maps in $\mathbb{R}^{n}$, Proc. Amer. Math. Soc. 104 (1988), 1111-1114.

3. P. Volkmann, Gewöhnliche Differentialgleichungen mit quasimonoton wachsenden Funktionen in topologischen Vektorräumen, Math. Z. 127 (1972), 157-164.

4. E. Zeidler, Nonlinear functional analysis and its applications, vol. I, Springer-Verlag, Berlin and New York, 1986.

FakUltät für Mathematik, Universität KarlsRUhe, Kaiserstrasse 12, D-7500 KarLSRUHE 1, GERMANY 